\title{
Malignant Brain Glioma
}

National Cancer Institute

\section{Source}

National Cancer Institute. Malignant Brain Glioma. NCI Thesaurus. Code C162993.

A grade III or IV glioma that arises from the brain. This category includes anaplastic astrocytoma, anaplastic ependymoma, anaplastic oligoastrocytoma, anaplastic oligodendrog lioma, anaplastic pleomorphic xanthoastrocytoma (all grade III gliomas), and glioblastoma (grade IV glioma). 